\title{
Encoding strategy and sensory and semantic interference
}

\author{
DOUGLAS L. NELSON, VALERIE S. REED and CATHY L. McEVOY \\ University of South Florida, Tampa, Florida $\$ 9620$
}

\begin{abstract}
Studies were designed to compare the effects of encoding strategies on two types of interference, sensory and semantic. Paired-associate lists were learned under instructional sets encouraging interactive imagery or repetitious rehearsal. Sensory interference was created by mispairing rhymes and semantic interference was produced by mispairing associatively related words. In control lists word pairs were unrelated. The results indicated that encoding strategy did not influence amounts of sensory and semantic interference. Mispairing rhymes produced the same amount of confusion as mispairing associates whether pairs were encoded through interaction imagery or through repetitious rehearsal. These effects were found with both short and long encoding intervals. The findings are inconsistent with the idea that encoding operations or strategies applied to word pairs completely determine the type of code activated for those pairs. Both sensory and semantic codes appear to be activated under each kind of encoding strategy.
\end{abstract}

Interference results when words serving as pairedassociate stimuli appear and sound similar, when they share graphic and phonemic features. This interference is remarkably tenacious in the sense that it is very difficult to eliminate experimentally. It is not eliminated by using concrete instead of abstract words, relatively long encoding and retrieval times, or by using semantically compatible word pairs (e.g., HAM EGGS, RAM HORN). It is not eliminated by simply training the subjects in using interactive verbal or imaginal mediation (Nelson \& Borden, 1973; Nelson \& Brooks, 1973; Nelson, Brooks, \& Borden, 1974; Nelson, Wheeler, \& Brooks, 1976). However, sensory interference is statistically nullified when long encoding intervals are combined with instructions encouraging the use of interactive semantic encoding (Nelson et al., 1976).

These findings can be interpreted within a levels-ofprocessing framework (Craik \& Lockhart, 1972; Craik \& Tulving, 1975; Nelson et al., 1976). The major as. sumption of these conceptualizations is that the sensory and semantic features of words undergo rapid perceptual and cognitive analyses, analyses that can result in the activation of qualitatively different types of codes. The sensory representation is theoretically linked to the physical stimulus, to its appearance and to its phonemic features. The semantic representation is linked to the significance of the stimulus, to its meaning and to its associative characteristics. Accordingly, in the pairedassociate task sensory interference is difficult to eliminate because the graphic and phonetic features of the

This research was supported by Grant MH 16360 to the first author from the National Institute of Mental Health. The authors are indebted to Richard Rickes and Patricia Shoemaker for their roles as experimenters in this series. Requests for reprints should be sent to: Douglas L. Nelson, Department of Psychology, University of South Florida, Tampa, Florida 33620. stimulus words must be processed before achieving semantic access. Thus, the tenacity of sensory interference should be related to conditions affecting the likelihood of semantic encoding. Such interference should be maintained when study time is limited and when instructions encourage sensory processing. Conversely, sensory interference might be eliminated under conditions that are highly favorable for developing semantic encodings, conditions that are apparently present when relatively long study intervals are combined with instructions that encourage the subjects to seek interactive and unifying relationships.

The initial aim of the present effort was simply to change the source of the sensory interference. In all earlier experiments, manipulations of similarity were confined to the stimuli. Therefore, Experiment 1 was first conceived as an extension of earlier work, an extension in which the source of the interference was located between the pairs of the paired-associate list. Thus, these pairs were either unrelated or incompatible. The incompatible lists consisted of rhymes that-were inappropriately paired, for example, PAIL-MAIL, JUNK-SKUNK, DITCH-WITCH, etc. became PAILSKUNK, JUNK-WITCH, DITCH-WELL, etc. This mispairing was expected to generate competition at test between the natural rhyme pairs and the pairs that had to be learned in the experiment. For example, correct functional contact with PAIL at test could lead to either "SKUNK" or "MAIL." Both kinds of lists were learned under instructional sets encouraging either imaginal or sensory encoding with either relatively short or long encoding intervals. The results were expected to parallel earlier findings, with the effects of rhyme incompatibility essentially eliminated through interactive imaginal encoding at the long study interval. However, this expectation was based on the assumption that this encoding strategy would eliminate decoding 
interference as well as it eliminated interference generated during stimulus contact processes in the earlier studies (Nelson et al., 1976).

The initial aim of this series was modified with Experiment 2 when it was decided to extend the interference manipulation to meaning. As in Experiment 1, encoding strategy and encoding time were varied. However, the incompatible lists consisted of mispaired associatively related words, for example, NECTAR-BEE, BABY-CRIB, CIRCLE-SQUARE, etc. became NECTARCRIB, BABY-SQUARE, CIRCLE-TRUNK, etc. At this point interest became primarily focused upon comparing the two types of interference, sensory and associative, under each instructional set. Thus, combining the two experiments allowed for direct comparisons of amounts of interference produced by similarities within each type of code. Experiment 3 replicated this manipulation within the confines of a single study.

These comparisons were expected to yield one of three interesting theoretical outcomes. First, the processing of the pairs might be completely controlled by the kind of mental activity required by the instructional sets (Craik \& Tulving, 1975; Jenkins, 1974; Kolers, 1975). Repetition instructions require overt rehearsal, a requirement that emphasizes the phonetic names for the items. Therefore, with repetition instructions a mispairing of similar name codes conceivably could generate more interference than a mispairing of related associative codes. An inverse relationship would be expected under imagery instructions. Interactive imaginal encoding presumably requires activation of meaning prior to developing imaginal representations. Therefore, with imagery instructions mispairing natural associative relationships should be more detrimental than mispairing natural sensory relationships. Taken together these interactions would suggest that instructional manipulations can produce an asymmetry in the effectiveness of the two types of codes, perhaps to the extent that only a single code is functional under a given encoding strategy. The second outcome is contingent upon the assumption that both sensory and associative codes are activated in performing this task. In this case, mispairing within either type of relationship should generate interference regardless of encoding instructions. While repetition emphasizes name codes, associative characteristics also may be activated. Similarly, while interaction imagery may require the processing of semantic and imaginal characteristics, the activation of sensory codes may necessarily precede such activation (Nelson, Wheeler, Borden, \& Brooks, 1974). Essentially, this result would be expressed in the statistical independence of encoding strategy and type of interference. While interaction imagery may reduce and possibly eliminate interference produced by mispairing, this reduction should not be tied to the specific type of interference. Finally, the third outcome represents a variation upon this result. Both types of codes may be activated in this task, but interaction imagery may eliminate inter- ference generated by sensory mispairing if encoding time is long. This result would be consistent with previous findings and would suggest that imaginal encoding also can eliminate sensory interference generated during processes associated with response retrieval.

\section{EXPERIMENTS 1 AND 2}

\section{Method}

Material. Each paired-associate list contained 12 pairs. The same words served as stimuli for both unrelated and incompatible lists and, therefore, these lists differed only in responses. Each unrelated list was constructed to avoid any type of relationship between stimuli and responses. Each incompatible list was derived by reassigning the responses of compatible pairings to other stimuli in the list, but simply switching responses for any two stimuli was not permitted (for details see Nelson \& Borden, 1973). In each experiment two different but apparently comparable lists were constructed for each unrelated and incompatible condition. Thus, for each experiment there were four different lists. All words were high in rated imagery value, averaging 5.85 according to the Colorado Imagery and Concreteness Norms.

Procedure. Subjects acquired single lists in individual sessions. On study trials each pair was presented for $2 \mathrm{sec}$ for half of the subjects and for $5 \mathrm{sec}$ for the remaining half. Test trials immediately followed study trials, and they were self-paced in that the next test stimulus was shown immediately after the subject's response. Test stimuli were pronounced aloud prior to response anticipation. All slides were shown by a Kodak Carousel slide projector, with the sequence of items independently randomized on study and on test trials. This randomization was changed for each subject in all conditions. The only restriction was that at least four tests had to intervene between a given pair and its test. Practice was continued for 20 trials or for 4 consecutive trials without error, whichever came first. Ninetyeight percent of the subjects achieved the performance criterion.

Just prior to reading the instructions for the task, each subject was shown the list of compatible pairs used to derive the incompatible list. Each subject was required to read the pairs aloud as shown, the ostensible purpose being to acquaint subjects with the presentation rate. In Experiment 1 the pairs rhymed, and they were presented twice; in Experiment 2 the pairs were associatively compatible, and they were shown once. The actual intent of this procedure was to increase the magnitude of interference attributable to incompatibility and to insure that these effects would be apparent on the very first trial. Originally, it was felt that a single reading trial on the associative pairs would generate as much interference as two reading trials on the rhymes. This phase was omitted in Experiment 3.

All subjects were told to "learn what words go together" during the study periods. For each experiment half of the subjects serving under each rate condition received interaction imagery instructions and the remaining half were given repetition instructions. In the one case, the instructions indicated that the most efficient way of learning the pairs was to construct a mental picture representing each word so that the images for each word interacted together. In the other case, the instructions suggested that greatest learning efficiency could be achieved by repeating the word pairs aloud as often as time allowed. Examples were provided for both instructional sets to emphasize the appropriate learning strategy. All subjects then learned the same practice list of three unrelated pairs before undertaking the experimental task.

Subjects. In each experiment there were two levels of pair relatedness, two presentation rates during study, two types of instructional sets, and two lists for each relatedness condition. Hence, there were a total of 16 between-subjects conditions in 
each study. In Experiment 1, the sensory incompatibility study, 8 subjects were assigned to each condition and list, making a total of 16 in each principal condition and 128 in the entire experiment. One male and one female collected all data, balanced with respect to conditions and lists. In Experiment 2, the associative incompatibility study, 9 subjects served in each treatment-list combination, providing a total of 18 in each of the major conditions and a total of 144 in the entire study. Three female experimenters collected the data.

Subjects were assigned to conditions-lists in blocks of 16 , with one subject from each condition per block. Assignment within blocks was determined by a table of random numbers. All subjects were selected from courses in introductory psychology and received course credit for participation.

\section{Results}

Mean errors per subject for each condition in the two experiments are shown in Table 1 . This table has been arranged to facilitate comparisons of type of interference for each rate of presentation. The first thing to note is that more errors were obtained with repetition than with imagery instructions, with fast as compared to slow rates, and with incompatible as compared to unrelated pairings. The next thing to note is that the amounts of interference produced by incompatibility appear to be very similar for both sensory and associative types of interference. That is, mispaired rhymes and mispaired associates appeared to produce approximately the same magnitude of interference under each condition of instruction and rate.

These observations were confirmed by the results of a four-factor analysis of variance. Instructions, presentation rate, and pair relatedness all represented significant sources at $p<.01$, with $\mathrm{Fs}(1,256)$ of $102.60,34.35$, and 48.62 and $\mathrm{MSe}=210.36$. Type of interference (experiments) was not reliable $(F=1.95)$. In fact, all of the interactions involving the type of interference factor were near unity. Thus, an equivalent amount of sensory and associative interference was produced, and the magnitude of this effect did not vary with either instructions or rate. The absence of any effect of type of interference was confirmed by a separate statistical analysis of the incompatible conditions.

The overall analysis did reveal two reliable interaction effects, Instructions by Rate $[F(1,256)=7.61]$ and

Table 1

Mean Errors Per Subject as a Function of Instructions, Relatedness, Presentation Rate, and Type of Interference

\begin{tabular}{|c|c|c|c|c|}
\hline \multirow[b]{3}{*}{$\begin{array}{c}\text { Type of } \\
\text { Interference }\end{array}$} & \multicolumn{4}{|c|}{ Instructions } \\
\hline & \multicolumn{2}{|c|}{ Imagery } & \multicolumn{2}{|c|}{ Repetition } \\
\hline & $\begin{array}{l}\text { Unre- } \\
\text { lated }\end{array}$ & $\begin{array}{l}\text { Incom- } \\
\text { patible }\end{array}$ & $\begin{array}{l}\text { Unre- } \\
\text { lated }\end{array}$ & $\begin{array}{l}\text { Incom- } \\
\text { patible }\end{array}$ \\
\hline \multicolumn{5}{|l|}{ 2-sec Rate } \\
\hline Sensory & 9.81 & 12.12 & 21.50 & 49.06 \\
\hline Associative & 9.50 & 14.44 & 24.06 & 42.33 \\
\hline \multicolumn{5}{|l|}{ 5-sec Rate } \\
\hline Sensory & 4.44 & 5.87 & 13.44 & 27.81 \\
\hline Associative & 2.22 & 4.06 & 7.78 & 20.00 \\
\hline Mean & 6.45 & 9.13 & 16.65 & 34.59 \\
\hline
\end{tabular}

Instructions by Pair Relatedness $[F(1,256)=18.83]$. The interaction with rate indicated that slowing the presentation rate produced a relatively greater reduction in errors under repetition than under imagery instructions. Mean errors under repetition instructions were 34.18 and 17.06 , respectively, at fast and slow rates; under imagery instructions, these values were 11.50 and 4.09. The interactions of Instructions by Pair Relatedness is displayed along the bottom row of the table. As can be seen, interference produced by pair incompatibility is greater when pairs are acquired through repetition than when they are learned through interactive imaginal encoding. Thus, the imagery mnemonic attenuated interference. However, as noted above, this attenuation was independent of the type of interference, sensory or associative. The $F$ for the Instructions by Pair Relatedness by Type of Interference interaction was less than unity.

The results of the overall analysis clearly indicated that the imagery mnemonic reduced interference produced by incompatibility. However, as indicated in the table, imagery instructions did not completely eliminate interference. Even under imagery instructions, more errors were made when the pairs were incompatible regardless of type of interference and rate. A separate statistical analysis of the imagery conditions confirmed this observation. Reliably more errors were produced when pairs were incompatible $[F(1,128)=6.62, p<.05]$ and when rate was fast $[\mathrm{F}(1,128)=50.77, \mathrm{p}<.01$, with MSe $=36.79]$. None of the remaining sources were reliable, including type of interference. A separate analysis of the repetition conditions resulted in an identical pattern of reliable sources.

The presence of interference under each encoding strategy also can be measured by the types of intrusions. A response intrusion occurred when a response from the list was given to the wrong stimulus. In the incompatible conditions these intrusions often took the form of saying the response naturally related to the stimulus. For example, if the pair was BOOT-HIVE, a response intrusion occurred when the subject said "SHOOT," a mispaired rhyme, or "SHOE," a mispaired associate. In order to assess the effects of instructional strategies upon sensory and associative interference presumably occurring during retrieval, response intrusions as proportions of total errors were tabulated for each subject and analyzed statistically. This analysis again indicated that type of interference had no reliable effect. The proportions of response intrusions were essentially equivalent under each encoding strategy for mispaired rhymes and mispaired associates. Furthermore, while interaction imagery reduced this type of retrieval interference at test, it did not reliably eliminate this competition. With imagery instructions the percentages of re sponse intrusions for incompatible and unrelated lists were reliably different, $19 \%$ and $10 \%$. With repetition instructions these values were $42 \%$ and $16 \%$.

A stimulus intrusion occurred when a stimulus from 
the list was given as a response. Again, if the pair was BOOT.HIVE, a stimulus intrusion occurred when the subject said "DIVE," a mispaired rhyme, or "BEE," a mispaired associate. These intrusions presumably also provide a measure of interference experienced at retrieval. These values also were converted to proportions of total errors in all conditions and subjected to statistical analysis. As with response intrusions, type of interference had no reliable effect. Proportionally more stimuli were intruded when pairs were incompatible $(26 \%)$ than when pairs were unrelated $(6 \%)$. Imaginal encoding, however, actually increased stimulus intrusions, especially at the slow rate. Under imagery instructions $23 \%$ of the total errors obtained at the slow rate were stimulus intrusions. At the fast rate this value was reduced to $12 \%$ and, under repetition instructions, rate had no effect with $15 \%$ of the errors classified as stimulus intrusions under each condition.

Finally, postexperimental interviews conducted on each pair in the list indicated that subjects utilized the encoding strategy specified by the instructions. Each pair was shown and each subject was asked to describe how he learned it. From these descriptions the experimenter classified the pairs as being elaboratively encoded or as rote learned. Thus, imaginal encoding was reportedly used for an average of 10.01 pairs learned under the imagery strategy, and this value dropped to 3.75 for pairs learned under the repetition strategy. These values did not change appreciably across other conditions in the experiment.

\section{EXPERIMENT 3}

The results of Experiments 1 and 2 indicated that encoding strategy and type of interference did not interact. The purpose of Experiment 3 was to compare the two types of interference in a single study and to assess the potential contribution of prior exposure to the compatible pairs as a possible factor in the maintenance of interference effects. Thus, this phase of the task was omitted in Experiment 3 . Instructions, relatedness, and type of interference were varied using only the 5-sec study rate.

\section{Method}

Materials and procedure. The lists, apparatus, instructions, and specific acquisition procedures were identical to those used in Experiments 1 and 2 . The only important and identifiable procedural differences consisted of omitting the prior exposure tasks and the 2 -sec exposure conditions.

Subjects. Sixteen subjects were assigned to each of the eight conditions formed by the 2 by 2 by 2 factorial, with eight assigned to each list. These subjects were drawn from the same sources and assigned to conditions in a manner similar to that used for the initial experiments. Two female experimenters collected half of the data for each condition and list.

\section{Results}

Table 2 displays mean errors per subject as a function of the major conditions. Examination of these values indicated two strong trends: Imagery instructions
Table 2

Mean Errors Per Subject as a Function of Instructions, Relatedness, and Type of Interference

\begin{tabular}{|c|c|c|c|c|}
\hline \multirow[b]{3}{*}{$\begin{array}{c}\text { Type of } \\
\text { Interference }\end{array}$} & \multicolumn{4}{|c|}{ Instructions } \\
\hline & \multicolumn{2}{|c|}{ Imagery } & \multicolumn{2}{|c|}{ Repetition } \\
\hline & $\begin{array}{l}\text { Unre- } \\
\text { lated }\end{array}$ & $\begin{array}{l}\text { Incom- } \\
\text { patible }\end{array}$ & $\begin{array}{l}\text { Unre- } \\
\text { lated }\end{array}$ & $\begin{array}{l}\text { Incom- } \\
\text { patible }\end{array}$ \\
\hline $\begin{array}{l}\text { Sensory } \\
\text { Associative }\end{array}$ & $\begin{array}{l}2.69 \\
2.12\end{array}$ & $\begin{array}{l}3.31 \\
4.75\end{array}$ & $\begin{array}{l}14.63 \\
10.06\end{array}$ & $\begin{array}{l}22.31 \\
18.88\end{array}$ \\
\hline
\end{tabular}

greatly facilitated performance in all conditions, but, more importantly, incompatibility produced by mispairing items generated interference in every comparison. The results of an analysis of variance of these data substantiated these trends. Instructions and pair relatedness were both significant sources at $\mathrm{p}<.05$, with $F_{s}(1,120)$ of 39.41 and 5.47 (MSe = 142.56). Type of interference and all interactions among these factors were not reliabie. Thus, as in Experiments 1 and 2 , neither sensory nor associative interterence was eliminated by interaction imagery instructions, even when pairs were presented at a slow 5 -sec study rate. The results of analyses of imagery reports, response and stimulus intrusions were generally consistent with those of the initial studies.

In order to evaluate the effects of prior exposure statistically, data displayed in the third and fourth rows of Table 1 were incorporated into a four-way analysis with the findings of Experiment 3 . Both sets of data were obtained at the 5 -sec study rate, with the only major difference being prior exposure. The results of this analysis indicated that reading the compatible pairings had no consistent effects, with all Fs involving prior exposure having values less than or near unity. Instructions and pair relatedness were reliable, as was the Instructions by Pair Relatedness interaction. The respective $F_{s}(1,248)$ were $104.90,23.68$, and 12.87 , with $\mathrm{MSe}=108.07$. The interaction reflected the fact that incompatibility had a greater disruptive effect under repetition instructions than under imagery instructions. Mean errors for unrelated and incompatible lists in the repetition conditions were 11.36 and 22.18 and, in the imagery conditions, these means were 2.85 and 4.48 . However, this interaction reflected an attenuation of interference, not its elimination. A separate statistical analysis of the imagery conditions indicated that pair relatedness remained as a significant source of variance, independent of both type of interference and prior exposure $[F(1,124)=6.95, p<.05, \quad M S e=12.70]$. Thus, even with imagery instructions, reliably more errors were obtained on the incompatible than on the unrelated pairings.

\section{DISCUSSION}

The findings of this series of experiments are clear. Mispairing rhymes or associates produces interference, interference that appears to be wholly independent of 
instructional set, amount of study time, and prior exposure to the compatible pairings. Thus, although repetition instructions require overt rehearsal and therefore stress the phonetic codes for the words, mispaired rhymes produced no more interference than mispaired associates under this instructional condition. Similarly, although interaction imagery instructions appear to stress the semantic and associative qualities of the words, mispaired associates produced interference that was equal to that of mispaired rhymes. Instructional manipulations of encoding strategies therefore failed to produce an asymmetry in the effectiveness of the two types of codes. This failure cannot be attributed to subjects' being unable or unwilling to abide by their instructions. Pair-by-pair interviews in which subjects described how they learned each pair suggest that the appropriate strategy was used for the vast majority of items. Thus, the outcome of these experiments would not support conceptualizations presuming that the activated representations of words directly and only correspond to the encoding operations or strategies applied to them during study (Craik \& Tulving, 1975; Jenkins, 1974; Kolers, 1975). At least in the pairedassociate task, overt rehearsal does not appear to activate only sensory codes, and imaginal processing does not appear to activate primarily semantic codes. Encoding strategy did affect performance in ways expected by this position in that operations stressing physical characteristics (repetition) produced more errors than operations stressing meaning (imagery). However, subjects appeared to remember more than what they did during encoding. Equivalent amounts of sensory and associative interference were generated under each encoding strategy. Regardless of what they did insofar as mental activity is concerned, the doing of it effectively activated both sensory and semantic codes. Thus, while the present findings reaffirm the importance of encoding strategy, they suggest that the activation of types of component attributes or features may be somewhat independent of these strategies.

The results of these experiments, however, are at odds with earlier findings, at least in one respect. Earlier findings indicated that sensory interference was statistically eliminated when long study times were combined with interaction imagery instructions (Nelson et al., 1976). In contrast, sensory interference in the present studies proved to be more tenacious. Identical instructions and identically long study times failed to eliminate it. Although there were many differences between the two sets of experiments, the primary one appears to be in the source or location of the interference. When confined to stimuli, the detrimental effects of sensory similarity are most likely limited to the stimulus recognition subprocess of paired-associate acquisition, to the subprocess involving contact during test between the external stimulus and its internal representation (Nelson, Brooks, \& Wheeler, 1975).
Semantic processing of the stimulus during study induced by elaborative encoding strategies apparently facilitates contact at test with the correct functional representation. For example, given sufficient time and semantic encoding of CANNON-BOTTLE, the likelihood of contacting the representation for CANNON and not CARTON or CAVERN is increased, increased to the point of circumventing the sensory interference (Nelson et al., 1976).

In the present series of experiments the source of the interference was completely different. There were no appreciable amounts of stimulus similarity, and, therefore, contact with the appropriate functional representation of the stimulus at test should not have been seriously impaired. However, response retrieval interference appeared to be great. Given that the list pair to be encoded was BOOT-HIVE, achieving correct contact with "BOOT" at test could lead to "HIVE," the correct response, or to "SHOOT," a response from the competing boot-shoot encoding, and one that represents an intrusion. Interactive imaginal encoding clearly reduced this source of interference relative to repetition encoding, but it did not eliminate it. Even with imagery instructions, response intrusions remained significantly higher in the incompatible relative to the unrelated lists. Finally, mispairing stimuli and responses produced still another potential source of retrieval interference. Even though "boot" may correctly activate "HIVE" at test, this response may be decoded as "DIVE," another stimulus from the list, an error in decoding that results in a stimulus intrusion. Interaction imagery instructions do not eliminate this kind of interference and, in fact, they increase it when encoding time is relatively long. Thus, when sensory interference is located among stimuli, there appears to be a single source of interference to be eliminated by elaborative encoding, the initial contact process between the external stimulus and its internal representation. When the interference is located across pairs in the list, it occurs primarily during two identifiable components of the retrieval process and, while elaborative encoding may reduce one of these components, it appears to exacerbate the other. As a result, interference produced by sensory similarity appears to be more tenacious when varied across pairs in the list than when limited only to stimuli.

\section{REFERENCES}

Craik, F. I., \& Lockhart, R. S. Levels of processing: A framework for memory research. Journal of Verbal Learning and Verbal Behavior, 1972, 11, 681-684.

Craik, F. I., \& Tulving, E. Depth of processing and the retention of words in episodic memory. Journal of Experimental Psychology: General, 1975, 104, 268-294.

Kolers. P. A. Memorial consequences of automatized encoding. Journal of Experimental Psychology: Human Learning and Memory, 1975, 1, 689-701.

Jenkins, J. J. Remember that old theory of memory? Well, forget it. American Psychologist, 1974, 29, 785-795. 
Nelson. D. L. \& Borden, R. C. Effect of meaning on processing phonetic features of words. Journal of Expertmental Psychology. 1973. 101. 373-375.

NEI SON. D L., \& Brooks, D. H. Independence of phonetic and imaginal features. Journal of Experiminial Fsichulogy, 1973. 97, 1-7.

Nelson, D. L., Brooks, D. H., \& Borden, R. C. Effects of turmal similarity: Phonetic. graphic or toth? Journal of Experimental Psychology, 1974, 103, 91-96.

Nelson D. L., Brooks. D. H.. \& Sheeler. J. W. Sensory and meaning features in stimulus recognition and associative retrieval. Jourmal of Experimental Psychology: Human Memory and Learning. 1975. 1. 711-719.
Nelson, D. L., WheEler, J. W., Borden, R. C., \& BRooks, D. H. Levels of processing and cuing: Sensory versus meaning features. Joumal of Experimental Psychology, 1974, 103, 971-977.

Nelson, D. L., WHEeler, J. W., \& Brooks, D. H. Meaning and the elimination of sensory interference. Journal of Experimental Psychology: Human Learning and Memory. 1976, 2. 95-102.

(Received for publication February 1, 1977; revision accepted May 13, 1977.) 